\title{
UNIFORM DISTRIBUTION OF TWO-TERM RECURRENCE SEQUENCES
}

\author{
WILLIAM YSLAS VELEZ
}

\begin{abstract}
Let $u_{0}, u_{1}, A, B$ be rational integers and for $n \geqslant 2$ define $u_{n}=A u_{n-1}$ $+B u_{n-2}$. The sequence $\left(u_{n}\right)$ is clearly periodic modulo $m$ and we say that $\left(u_{n}\right)$ is uniformly distributed modulo $m$ if for every $s$, every residue modulo $m$ occurs the same number of times in the sequence of residues $u_{s}, u_{s+1}, \ldots, u_{s+N-1}$, where $N$ is the period of $\left(u_{n}\right)$ modulo $m$. If $\left(u_{n}\right)$ is uniformly distributed modulo $m$ then $m$ divides $N$, so we write $N=m f$. Several authors have characterized those $m$ for which $\left(u_{n}\right)$ is uniformly distributed modulo $m$. In fact in this paper we will show that a much stronger property holds when $m=p^{k}, p$ a prime. Namely, if $\left(u_{n}\right)$ is uniformly distributed modulo $p^{k}$ with period $p^{k} f$, then every residue modulo $p^{k}$ appears exactly once in the sequence $u_{s}, u_{s+f}, \ldots, u_{s+\left(p^{k}-1\right) f}$, for every $s$. We also characterize those composite $m$ for which this more stringent property holds.
\end{abstract}

Let $u_{0}, u_{1}, A, B$ be rational integers and define, for $n \geqslant 2, u_{n}=A u_{n-1}+B u_{n-2}$. The sequence of integers $\left(u_{n}\right)$ thus obtained is said to be a two-termed linear recurrence sequence. If $m$ is a positive integer then the sequence $\left(u_{n}\right)$ considered modulo $m$ is clearly periodic.

Definition. The sequence $\left(u_{n}\right)$ is said to be uniformly distributed modulo $m$ (henceforth denoted by $\mathrm{UD}(\bmod m)$ ) if every residue modulo $m$ occurs the same number of times in any period. That is, if $N$ is the period of $\left(u_{n}\right)$ modulo $m$, then for every $s$, every residue modulo $m$ appears the same number of times among the residues $\left\{u_{s}, u_{s+1}, \ldots, u_{s+N-1}\right\}$.

Those $m$ for which $\left(u_{n}\right)$ is $\mathrm{UD}(\bmod m)$ have been determined by several authors and recently Narkiewicz [2] has collected these results together. We shall use the notation and results of Chapter 3 of [2] throughout this paper.

In order to state this characterization we begin by developing some terminology. Given $\left(u_{n}\right)$, let $D=A^{2}+4 B$ be the discriminant of $x^{2}-A x-B$. We can express $u_{n}$ in terms of the roots of the quadratic in the following way (see Chapter 3 of [2]).

Case I, $D=0$. Then $u_{n}=\left(c_{0}+c_{1} n\right)(A / 2)^{n}$, for $n \geqslant 0$ and $c_{0}=u_{0}, c_{1}=$ $\left(2 u_{1}-A u_{0}\right) A^{-1}$.

Case II, $D \neq 0$. Then $u_{n}=c_{0}((A+\sqrt{D}) / 2)^{n}+c_{1}((A-\sqrt{D}) / 2)^{n}$, where $c_{0}=$ $\left(u_{0} \sqrt{D}+\left(2 u_{1}-A u_{0}\right)\right) / 2 \sqrt{D}, c_{1}=\left(u_{0} \sqrt{D}-\left(2 u_{1}-A u_{0}\right)\right) / 2 \sqrt{D}$.

Received by the editors August 5, 1985.

1980 Mathematics Subject Classification (1985 Revision). Primary 10A35.

The author was supported in part by National Science Foundation Grant \# PRM 82-13783.

(C)1987 American Mathematical Society $0002-9947 / 87 \$ 1.00+\$ .25$ per page 
THEOREM A. The sequence $\left(u_{n}\right)$ is $\mathrm{UD}(\bmod m)$ iff the following hold:

(i) If a prime $p$ divides $m$ then $p$ divides $D$ and $p+B$.

(ii) If $p \geqslant 3$ then $p+2 u_{1}-A u_{0}$.

(iii) If $p=3$ and $9 \mid m$ then $D \not \equiv 6(\bmod 9)$.

(iv) If $p=2$ then $u_{0}, u_{1}$ have opposite parity and if $4 \mid m$ then $A \equiv 2(\bmod 4)$ and $B \equiv 3(\bmod 4)$.

If $\left(u_{n}\right)$ is $\mathrm{UD}(\bmod m)$ then it is obvious that the period of $\left(u_{n}\right)$ modulo $m$ is divisible by $m$. Henceforth let us denote this period by $m f$.

If one specializes the above to the Fibonacci sequence, $u_{0}=0, u_{1}=A=B=1$, then $\left(u_{n}\right)$ is $\mathrm{UD}(\bmod m)$ iff $m=5^{k}$ and the period is $5^{k} \cdot 4$. For this sequence Erlebach and Vélez [1] discovered that in fact $\left(u_{n}\right)$ satisfies an even more stringent property modulo $5^{k}$, namely, for every $s$, every residue modulo $5^{k}$ occurs exactly once in the sequence $u_{s}, u_{s+4}, \ldots, u_{s+\left(5^{k}-1\right) 4}$.

In this paper we shall see that this same type of distribution holds for the more general cases of $\mathrm{UD}\left(\bmod p^{k}\right)$ and we shall also characterize those composite $m$ for which the above property holds. With this in mind we make the following definition.

Definition. Let $\left(u_{n}\right)$ be $\mathrm{UD}(\bmod m)$ with period $m f$. Then we say that $\left(u_{n}\right)$ is $f$-UD $(\bmod m)$ if for every $s$ every residue modulo $m$ occurs exactly once in the sequence $u_{s}, u_{s+f}, \ldots, u_{s+(m-1) f}$.

As mentioned above we shall prove the following.

THEOREM B. The sequence $\left(u_{n}\right)$ is $\mathrm{UD}\left(\bmod p^{k}\right)$ with period $p^{k} f$ iff $\left(u_{n}\right)$ is $f$-UD $\left(\bmod p^{k}\right)$. Furthermore, $f=1$ if $p=2$ otherwise it is the multiplicative order of $A / 2$ modulo $p$.

It is obvious that if $\left(u_{n}\right)$ is $f-\mathrm{UD}(\bmod m)$ then $\left(u_{n}\right)$ is $\mathrm{UD}(\bmod m)$. Thus we only have to prove one direction. What we shall actually prove is that if $\left(u_{n}\right)$ and $p$ satisfy conditions (i)-(iv) then $\left(u_{n}\right)$ is $f-\mathrm{UD}\left(\bmod p^{k}\right)$.

The method of proof will be as follows. We shall expand $((A \pm \sqrt{D}) / 2)^{n}$ using the binomial theorem and reduce the expression in the appropriate residue system. Before launching into a proof we must first deal with some technical matters.

For a prime $p$ let $\nu_{p}(a)$ denote the exact power of $p$ that divides the integer $a$. For a rational number $a / b$ we set $\nu_{p}(a / b)=\nu_{p}(a)-\nu_{p}(b)$.

Lemma 1. Suppose that conditions (i)-(iv) of $A$ are satisfied, $D \neq 0$ and $j \geqslant 1$.

If $p=2, \nu_{2}\left((2 j+1) ! A^{2 j}\right)=\nu_{2}\left((2 j) ! A^{2 j}\right)<4 j \leqslant \nu_{2}\left(D^{j}\right)$.

If $p \geqslant 5$ or $\nu_{p}(D)>1$, then $\nu_{p}\left((2 j+1) ! A^{2 j}\right)<\nu_{p}\left(D^{j}\right)$.

If $p=3$ and $\nu_{3}(D)=1$, then

$$
\nu_{3}\left((2 j+1) ! A^{2 j}\right) \leqslant j=\nu_{3}\left(D^{j}\right) .
$$

Further $\nu_{3}\left((2 j+1) ! A^{2 j}\right)=j$ iff $2 j+1$ is a power of 3 .

Proof. It is well known that

$$
\nu_{p}((2 j+1) !)=\sum_{h=1}^{\infty}\left[\frac{2 j+1}{p^{h}}\right],
$$


where [ ] denotes the greatest integer function. Let $s$ be defined by $p^{s} \leqslant 2 j+1<$ $p^{s+1}$.

If $p=2$ and $k \geqslant 2$ then since $A \equiv 2(\bmod 4), B \equiv 3(\bmod 4)$ we see that $\nu_{2}(D) \geqslant 4$ and $\nu_{2}\left(A^{2 j}\right)=2 j$, so

$$
\begin{aligned}
\nu_{2}\left((2 j+1) ! A^{2 j}\right) & =2 j+\nu_{2}((2 j) !)=2 j+\sum_{h=1}^{s}\left[\frac{2 j}{2^{h}}\right] \\
& \leqslant 2 j+\sum_{h=1}^{s} \frac{2 j}{2^{h}}=2 j+2 j\left(1-2^{-s}\right) .
\end{aligned}
$$

However, since the left-hand side is an integer we have that

$$
\nu_{2}\left((2 j) ! A^{2 j}\right)=\nu_{2}\left((2 j+1) ! A^{2 j}\right)<4 j \leqslant \nu_{2}\left(D^{s}\right) .
$$

The remaining cases follow the same pattern.

LEMMA 2. Suppose that $u_{0}, u_{1}$ satisfy conditions (i)-(iv). If we replace $u_{0}, u_{1}$ by $u_{s}$, $u_{s+1}$ in (i)-(iv), then $u_{s}, u_{s+1}$ also satisfy conditions (i)-(iv).

Proof. It is obvious that if $u_{0}, u_{1}$ have opposite parity then $u_{s}, u_{s+1}$ also have opposite parity. Thus it only remains to show that $p+\left(2 u_{s+1}-A u_{s}\right)$, where $p$ is an odd prime satisfying $p \mid D$ and $p+B$. From this it follows that $p+A$ and $(A / 2)^{2} \equiv$ $-2 B(\bmod p)$.

Suppose that $p+2 u_{k}-A u_{k-1}$ and consider $2 u_{k+1}-A u_{k}$. Since $u_{k+1}=A u_{k}+$ $B u_{k-1}$, we have that

$$
\begin{aligned}
2 u_{k+1}-A u_{k} & =A u_{k}+2 B u_{k-1} \equiv A u_{k}-\left(A^{2} / 2\right) u_{k-1} \\
& \equiv(A / 2)\left(2 u_{k}-A u_{k-1}\right) \quad(\bmod p),
\end{aligned}
$$

so $p+2 u_{k+1}-A u_{k}$.

The formulas appearing in Cases I and II are rather cumbersome. The next two lemmas will allow us to reduce the analysis to the case where $u_{0}=0$ and $u_{1}=1$.

Lemma 3. Suppose that $\left(u_{n}\right)$ and $p$ satisfy (i)-(iv). Given any $k$ there exists an $n$ such that $\nu_{p}\left(u_{n}\right) \geqslant k$.

Proof. Case I: $u_{n}=\left(c_{0}+c_{1} n\right)(A / 2)^{n}$. From the assumptions we see that $\left(p, c_{1}\right)=(p, A / 2)=1$, so we can easily solve the linear congruence $c_{0}+c_{1} n \equiv 0$ $\left(\bmod p^{k}\right)$.

Case II. By applying the binomial theorem to $(A \pm \sqrt{D})^{n}$, we obtain

$$
\begin{aligned}
u_{n}=\left(\frac{A}{2}\right)^{n}\left[u_{0}\left(1+\left(\begin{array}{c}
n \\
2
\end{array}\right) A^{-2} D+\left(\begin{array}{c}
n \\
4
\end{array}\right) A^{-4} D^{2}+\cdots\right)\right. & \\
& \left.+\left(2 u_{1}-A u_{0}\right)\left(\left(\begin{array}{c}
n \\
1
\end{array}\right) A^{-1}+\left(\begin{array}{c}
n \\
3
\end{array}\right) A^{-3} D+\left(\begin{array}{c}
n \\
5
\end{array}\right) A^{-5} D^{2}+\cdots\right)\right] .
\end{aligned}
$$

First of all observe that by 1 all of the expressions involving the binomial coefficients are integral at $p$.

Let us write $n$ in the form $n=p^{k-1} m$, where $k \geqslant 1$ and $m$ is to be determined later. If $\nu_{p}\left(D^{j} / A^{2 j+1}(2 j+1) !\right)>0$ or $\nu_{p}\left(D^{j} / A^{2 j}(2 j) !\right)>0$, then

$$
\nu_{p}\left(\left(\begin{array}{c}
p^{k-1} m \\
2 j+1
\end{array}\right) A^{-2 j-1} D^{j}\right) \geqslant k \text { and } \nu_{p}\left(\left(\begin{array}{c}
p^{k-1} m \\
2 j
\end{array}\right) A^{-2 j} D^{j}\right) \geqslant k \text {. }
$$


Thus from Lemma 1 it follows that for $n=p^{k-1} m$,

$$
u_{0}\left[1+\left(\begin{array}{l}
n \\
2
\end{array}\right) A^{-2} D+\left(\begin{array}{l}
n \\
4
\end{array}\right) A^{-4} D^{2}+\cdots\right] \equiv u_{0} \quad\left(\bmod p^{k}\right) .
$$

Further, if $p \geqslant 5$ or $\nu_{p}(D)>1$, then from Lemma 1 we have that

$$
u_{n} \equiv(A / 2)^{n}\left[\dot{u}_{0}+\left(2 u_{1}-A u_{0}\right) A^{-1} p^{k-1} m\right]\left(\bmod p^{k}\right) .
$$

We will now induct on $k$. If $k=1$, then if $p$ is odd

$$
u_{m} \equiv(A / 2)^{m}\left[u_{0}+\left(2 u_{1}-A u_{0}\right) A^{-1} m\right] \equiv 0 \quad(\bmod p)
$$

has a solution for some $m$ since $\left(\left(2 u_{1}-A u_{0}\right), p\right)=1$. If $p=2$, then $u_{0}, u_{1}$ have opposite parity, so at least one of $u_{0}, u_{1}$, is divisible by 2 .

Thus, assume there is an $s$ for which $u_{s} \equiv 0\left(\bmod p^{k-1}\right)$. By Lemma 2 we may assume that $u_{0} \equiv 0\left(\bmod p^{k-1}\right)$. So let $u_{0}=p^{k-1} v$. Then

$$
u_{n} \equiv(A / 2)^{n}\left[p^{k-1} v+\left(2 u_{1}-A u_{0}\right) A^{-1} p^{k-1} m\right] \equiv 0 \quad\left(\bmod p^{k}\right)
$$

iff

$$
u_{n} p^{-k+1} \equiv(A / 2)^{n}\left[v+\left(2 u_{1}-A u_{0}\right) A^{-1} m\right] \equiv 0 \quad(\bmod p),
$$

which clearly has a solution for some $m$. Thus the lemma is true if $p \geqslant 5$ or $\nu_{p}(D)>1$.

Let us now consider $p=3$ and $\nu_{3}(D)=1$. Then if $j \geqslant 2$,

$$
\nu_{3}\left(\left(p^{k-1} m\right) ! /\left(p^{k-1} m-(2 j+1)\right)\right) \geqslant k
$$

so

$$
u_{n} \equiv(A / 2)^{n}\left[u_{0}+\left(2 u_{1}-A u_{0}\right)\left(A^{-1} 3^{k-1} m+\left(\begin{array}{c}
n \\
3
\end{array}\right) A^{-3} D\right)\right]\left(\bmod 3^{k}\right) .
$$

Again we induct on $k$. Since $\left(u_{n}\right)$ and 3 satisfy (i)-(iv) and $\nu_{3}(D)=1$, we have that $D \equiv 3(\bmod 9)$, so $D / 3 \equiv 1(\bmod 3)$.

For $k=1$, we have that $n=3^{1-1} m=m$,

$$
\begin{aligned}
u_{m} & \equiv(A / 2)^{m}\left[u_{0}+\left(2 u_{1}-A u_{0}\right)\left(A^{-1} m+m(m-1)(m-2) A^{-3}(D / 3) / 2\right)\right] \\
& \equiv(A / 2)^{m}\left[u_{0}+\left(2 u_{1}-A u_{0}\right)\left(A^{-1} m\right)\right] \quad(\bmod 3),
\end{aligned}
$$

since $m(m-1)(m-2) \equiv 0(\bmod 3)$. Since $\left(2 u_{1}-A u_{0}, 3\right)=1$, there is certainly an $m$ for which $u_{m} \equiv 0(\bmod 3)$.

Thus by induction we may assume that there is an $s$ for which $u_{s} \equiv 0\left(\bmod 3^{k-1}\right)$ and as before we may assume that $s=0$ and $u_{0}=3^{k-1} v$. Thus

$$
\begin{aligned}
u_{n} 3^{-k+1} & \equiv(A / 2)^{n}\left[v+\left(2 u_{1}-3^{k-1} m A\right)\left(A^{-1} m+m\left(3^{k-1} m-1\right)\right.\right. \\
& \equiv(A / 2)^{n}\left[v+2 u_{1}\left(A^{-1} m+m(-1)(-2) A^{-3} / 2\right)\right] \\
& \equiv(A / 2)^{n}\left[v+2 u_{1} A^{-1} m\left(1+A^{-2}\right)\right] \\
& \equiv(A / 2)^{n}\left[v+u_{1} A^{-1} m\right](\bmod 3), \quad \text { since } A^{2} \equiv 1(\bmod 3) .
\end{aligned}
$$


Since $3 \mid u_{0}$ and $3+\left(2 u_{1}-A u_{0}\right)$, we have that $\nu_{3}\left(u_{1} A^{-1}\right)=0$ so there is an $m$ for which $v+u_{1} A^{-1} m \equiv 0(\bmod 3)$ so the lemma is proven.

REMARK. The reader will note that if $D / 3 \equiv 2(\bmod 3)$ then $1+(D / 3) A^{-2} \equiv 0$ $(\bmod 3)$ and the induction fails to go through.

COROllary 4. Suppose that $\left(u_{n}\right)$ and p satisfy (i)-(iv). Then if we are considering the sequence $\left(u_{n}\right)$ modulo $p^{k}$ we may assume that $u_{0}=0, u_{1}=1$ and $u_{n}$ modulo $p^{k}$ is given by the formulas:

Case I. $u_{n} \equiv(A / 2)^{n-1} n\left(\bmod p^{k}\right)$.

Case II. $u_{n} \equiv(A / 2)^{n}\left(\left(\begin{array}{l}n \\ 1\end{array}\right)+\left(\begin{array}{c}n \\ 3\end{array}\right) A^{-2} D+\left(\begin{array}{c}n \\ 5\end{array}\right) A^{-4} D^{2}+\cdots\right)\left(\bmod p^{k}\right)$.

Proof. From the previous lemmas we may assume that $u_{0} \equiv 0\left(\bmod p^{k}\right)$. Since $p+2 u_{1}-A u_{0}$ if $p$ is odd, this implies that $p+u_{1}$. Also if $p=2$ then $u_{0}, u_{1}$ having opposite parity yields that $2+u_{1}$. Thus in all cases $p+u_{1}$. If we multiply $u_{n}$ by $u_{1}^{-1}$ then $\left(u_{1}^{-1} u_{n}\right)$ satisfy (i)-(iv).

Now that we have these preliminaries out of the way we can begin to obtain information about the periods of uniformly distributed sequences.

LEMMA 5. Let the order of $A / 2$ modulo $p$ be $f$. If $\left(u_{n}\right)$ is $\mathrm{UD}\left(\bmod p^{k}\right)$ its period is $p^{k} f$.

Proof. Without loss of generality we may assume that $u_{0}=0$ and $u_{1}=1$.

Case I. Since $u_{n} \equiv(A / 2)^{n-1} n\left(\bmod p^{k}\right)$, we have that the order of $A / 2$ modulo $p^{k}$ is $p^{j} f$, where $j \leqslant k-1$ and the period of $n$ modulo $p^{k}$ is $p^{k}$, so the assertion follows.

Case II. Then $u_{n} \equiv(A / 2)^{n-1} B(n)\left(\bmod p^{k}\right)$, where $B(n)=\left(\begin{array}{l}n \\ 1\end{array}\right)+\left(\begin{array}{l}n \\ 3\end{array}\right) A^{-2} D+$ $\left(\begin{array}{c}n \\ 5\end{array}\right) A^{-4} D^{2}+\cdots$, where of course the sum is finite.

For $k=1$, the period of $(A / 2)^{n-1}$ is $f$ and the period of $B(n)$ is $p$, thus the period of $u_{n}$ is $p f$.

For general $k$ we have that the period of $\left(u_{n}\right)$ modulo $p^{k}$ is $p^{k} h$, for some $h$. As before $(A / 2)$ has period $f p^{j}$, where $j \leqslant k-1$, so $f \mid h$. It is also clear that $\left(\begin{array}{c}n \\ 2 j+1\end{array}\right) A^{-2 j} D^{j}$ has period a divisor of $p^{k}$. Thus the period of $(A / 2)^{n-1} B(n)$ divides $p^{k} f$, so the period is $p^{k} f$.

The determination of $f-\mathrm{UD}\left(\bmod p^{k}\right)$ involves the analysis of $(A / 2)^{n-1} B(n)$ $\left(\bmod p^{k}\right)$. A useful technical result will be the following.

LEMMA 6. Let $n=m+p^{k-1} m_{1}$. If either (a) $p \geqslant 5$, (b) $\nu_{p}(D)>1$, or (c) $p=3$, $\nu_{3}(D)=1$ and $j \geqslant 2$, then

$$
\left(\begin{array}{c}
n \\
2 j+1
\end{array}\right) A^{-2 j} D^{j} \equiv\left(\begin{array}{c}
m \\
2 j+1
\end{array}\right) A^{-2 j} D^{j} \quad\left(\bmod p^{k}\right) .
$$

Proof. If $\nu_{p}\left(D^{j} / A^{2 j}(2 j+1)\right.$ !) $>0$ then the result follows, and this occurs if $p \geqslant 5$ or $\nu_{p}(D)>1$ or $p=3, \nu_{p}(D)=1$ and $2 j+1$ is not a power of 3 , by Lemma 1.

Thus, let us consider $p=3, \nu_{p}(D)=1$ and $2 j+1=3^{r}$. Then

$$
\alpha=D^{j} A^{-2 j} /(2 j+1) \text { ! }
$$


is integral at 3. Set $\lambda=n(n-1) \cdots(n-2 j)$. A factor of $\lambda$ is of the form $n-i$, where $i \in\{0,1, \ldots, 2 j\}$. Thus write $\lambda=(n-i) \lambda_{i}$. Now

$$
\lambda=\left(m-i+p^{k-1} m_{1}\right) \lambda_{i}
$$

and $3 \mid \lambda_{i}$ since $2 j \geqslant 8$ (recall $2 j=3^{r}, r \geqslant 2$ ), so $\lambda$ is the product of at least 8 consecutive integers. Thus $\lambda \equiv(m-i) \lambda_{i}$ and the result follows.

We can now prove the main result on $f-\mathrm{UD}\left(\bmod p^{k}\right)$.

THEOREM 7. Let $\left(u_{n}\right)$ and $p^{k}$ satisfy conditions (i)-(iv). If $A / 2$ has order $f$ modulo $p$, then $\left(u_{n}\right)$ is $f-\mathrm{UD}\left(\bmod p^{k}\right)$.

Proof. We want to show that for any $s, u_{s}, u_{s+f}, \ldots, u_{s+f\left(p^{k}-1\right)}$ are all distinct residues modulo $p^{k}$. Let us first prove the assertion for $k=1$. If $p=2$ the assertion is obvious.

In either Case I or II we have that $u_{n} \equiv(A / 2)^{n-1} n(\bmod p)$. Thus for $n=s+a f$, $a \in\{0,1, \ldots, p-1\}$ we have that

$$
u_{n} \equiv(A / 2)^{s-1}(A / 2)^{a f}(s+a f) \equiv(A / 2)^{s-1}(s+a f) \quad(\bmod p),
$$

since $(A / 2)^{f} \equiv 1(\bmod p)$. Since $f \mid p-1, s+$ af runs through the distinct residues modulo $p$ as $a$ runs through $\{0,1, \ldots, p-1\}$. Thus we have the result is true for $k=1$ and now let us assume that the result is true for $k-1$.

For $k>1$ and $a \in\left\{0,1, \ldots, p^{k}-1\right\}$ let us write $a$ in the form $a=b+c p^{k-1}$, where $b \in\left\{0,1, \ldots, p^{k-1}-1\right\}, c \in\{0,1, \ldots, p-1\}$. Thus

$$
\begin{aligned}
u_{s+a f} & \equiv(A / 2)^{s-1}(A / 2)^{b f}(A / 2)^{c f p^{k-1}} B(s+a f) \\
& \equiv(A / 2)^{s-1}(A / 2)^{b f} B(s+a f) \quad\left(\bmod p^{k}\right) .
\end{aligned}
$$

If we consider $u_{s+a f}$ modulo $p^{k-1}$, then $B(s+a f) \equiv B(s+b f) \bmod \left(p^{k-1}\right)$, so $u_{s+a f} \equiv(A / 2)^{s-1}(A / 2)^{b f} B(s+b f)\left(\bmod p^{k-1}\right)$. Thus the induction hypothesis yields that as $b$ ranges through the set $\left\{0,1, \ldots, p^{k-1}\right\}$, these are all distinct modulo $p^{k-1}$.

Thus let us now let $b$ be fixed and let $c$ range through the set $\{0,1, \ldots, p-1\}$. So we have $u_{s+a f} \equiv(A / 2)^{s-1}(A / 2)^{b f} B\left(s+b f+c f p^{k-1}\right)\left(\bmod p^{k}\right)$. So these are all incongruent iff $B\left(s+b f+c f p^{k-1}\right)\left(\bmod p^{k}\right)$ are all incongruent.

By Lemma 6 we have that if $p \geqslant 5$ or $\nu_{p}(D)>0$, then

$$
B(s+a f) \equiv\left(\begin{array}{c}
s+b f+c f p^{k-1} \\
1
\end{array}\right)+C(s, b) \quad\left(\bmod p^{k}\right),
$$

where $C(s, b)$ depends only on $b$ and $s$ and not on $c$. Thus $B(s+a f)$ are clearly all incongruent as $c$ ranges through the set $\{0,1, \ldots, p-1\}$.

If $p=3$ and $\nu_{3}(D)=1$, then

$$
B(s+a f) \equiv\left(\begin{array}{c}
s+b f+c 3^{k-1} \\
1
\end{array}\right)+\left(\begin{array}{c}
s+b f+c 3^{k-1} \\
3
\end{array}\right) A^{-2} D+C_{1}(s, b)\left(\bmod 3^{k}\right),
$$

where again $C_{1}(s, b)$ depends only on $b$ and $s$ and not on $c$. Set $B_{1}(s+a f) \equiv$ $B(s+a f)-C_{1}(s, b)$, and we shall prove the assertion for $B_{1}(s+a f)$. 
If $k \geqslant 3$ then condition (iii) gives that $D \not \equiv 6(\bmod 9)$. Since we are also assuming that $\nu_{3}(D)=1$, this implies that $D \equiv 3(\bmod 9)$, so $A^{-2} D / 3 \equiv 1(\bmod 3)$.

Let $c_{1}=c f$, then since $(f, 3)=1, c_{1}$ ranges over the residue system $\{0,1,2\}$ as $c$ ranges over the same residue system. Set $m=s+b f$. Then

$$
\begin{aligned}
& B_{1}(s+a f) \equiv m+c_{1} 3^{k-1}+\left(m+c_{1} 3^{k-1}\right)\left(m-1+c_{1} 3^{k-1}\right) \\
& \equiv m+c_{1} 3^{k-1}+\left[m(m-1)(m-2)+c_{1} 3^{k-1}\right. \\
& \equiv \\
& \cdot(m(m-1)+m(m-2)+(m-1)(m-2))] A^{-2} D /(2 \cdot 3) \\
& \equiv m+c_{1} 3^{k-1}+\left[m(m-1)(m-2)+2 c_{1} 3^{k-1}\right] A^{-2} D /(2 \cdot 3) \\
& \equiv C_{2}(s, b)+c_{1} 3^{k-1}\left(\bmod 3^{k}\right),
\end{aligned}
$$

where $C_{2}(s, b)$ collects together all those terms which do not contain $c_{1}$.

Thus it is obvious that $B_{1}(s+a f)$ are all distinct as $c_{1}$ runs through the residue system module 3 .

We have proved Theorem B and in so doing we have proven A for the case $m$ a prime power. It has already been observed that if $\left(u_{n}\right)$ is $\operatorname{UD}\left(\bmod p^{k}\right)$ for every $p^{k}$ dividing $m, p$ a prime, then $\left(u_{n}\right)$ is $\mathrm{UD}(\bmod m)$. We shall not prove this result again (though at the end of this paper we shall make some remarks about a different proof), rather we shall assume the validity of $A$ and characterize when $\left(u_{n}\right)$ is $f-\mathrm{UD}(\bmod m)$.

Let $m=P_{1} \cdots P_{r}$, where each $P_{i}$ is a prime power, $\left(P_{i}, P_{j}\right)=1$ if $i \neq j$. We shall assume that $u_{n}$ is $\mathrm{UD}\left(\bmod P_{i}\right)$, with period $P_{i} f_{i}$, for each $i$ (thus by Theorem $\mathrm{B}$, $\left(u_{n}\right)$ is $\left.f_{i}-\mathrm{UD}\left(\bmod P_{i}\right)\right)$.

The period of $\left(u_{n}\right)$ modulo $m$ is the 1.c.m. $\left\{P_{1} f_{1}, \ldots, P_{r} f_{r}\right\}$, which we shall write as $m f$.

We shall need the following technical result to arrive at this characterization.

LEMmA 8. Suppose that $\left(u_{n}\right)$ is $f-\mathrm{UD}(\bmod m)$, then $u_{s}, u_{s+h f}, u_{s+2 h f}, \ldots$, $u_{s+(m-1) h f}$ are all distinct modulo $m$ iff $(h, m)=1$.

Proof. If $(h, m)=1$ then $0, h, \ldots,(m-1) h$ are all distinct modulo $m$. Given $j$, let $k(j)$ be the least residue between 0 and $m$ congruent to $j h$ modulo $m$. Thus $j h f \equiv k(j) f+l(j) m f$. However, $\left(u_{n}\right)$ has period $m f$ modulo $m$, so $u_{s+j h f} \equiv u_{s+k(j) f}$ $(\bmod m)$ and since $u_{s}, u_{s+f}, \ldots, u_{s+(m-1) f}$ are all distinct modulo $m$ the result follows.

Conversely, if $(h, m) \neq 1$, there exist $0<i<j<m$ such that $i h \equiv j h(\bmod m)$, so $i h f=j h f+\operatorname{lmf}$ and $u_{s+i h f} \equiv u_{s+j h f}(\bmod m)$. Thus $u_{s}, u_{s+h f}, \ldots, u_{s+(m-1) h f}$ are not all distinct modulo $m$.

THEOREM 9. Suppose that $m=P_{1} \cdots P_{r}$ and that $\left(u_{n}\right)$ is $f_{i}-\mathrm{UD}\left(\bmod P_{i}\right), i=$ $1, \ldots, r$. Let 1.c.m. $\left\{P_{1} f_{1}, P_{2} f_{2}, \ldots, P_{r} f_{r}\right\}=m f$. Then $\left(u_{n}\right)$ is $f-\mathrm{UD}(\bmod m)$ iff $(f, m)=1$. 
Proof. Let $p_{i}$ be the prime corresponding to $P_{i}$, that is, $P_{i}$ equals $p_{i}$ to some positive exponent. We shall number the $P_{i}$ so that $p_{1}<p_{2}<\cdots<p_{r}$.

Assume that $(f, m)=1$. We shall prove the theorem by inducting on $r$. For $r=1$ this is Theorem B. Set $F=P_{1}, L=P_{2} \cdots P_{r}$ and consider the matrix

$$
A=\left[\begin{array}{cccc}
u_{s} & u_{s+f} & \cdots & u_{s+(F-1) f} \\
u_{s+F f} & u_{s+(F+1) f} & \cdots & u_{s+(2 F-1) f} \\
\vdots & & & \\
u_{s+(L-1) F f} & u_{s+((L-1) F+1) f} & \cdots & u_{s+(m-1) f}
\end{array}\right]
$$

Since $P_{1} f_{1} \mid P_{1} \cdots P_{r} f$, we have that $f_{1} \mid P_{2} \cdots P_{r} f$. However, $f_{1} \mid p_{1}-1$ since $f_{1}$ is the multiplicative order of $A / 2$ modulo $p_{1}$ if $p_{1}$ is odd, otherwise $f_{1}=1$. Since $p_{1}<p_{2}<\cdots<p_{r}$, this implies that $\left(f_{1}, P_{2} \cdots P_{r}\right)=1$, so $f_{1} \mid f$, and we write $f=h_{1} f_{1}$. By assumption, $\left(f, P_{1}\right)=1$, so $\left(h_{1}, P_{1}\right)=1$ and from Lemma 8 we may conclude that each row of the matrix $A$ represents all of the distinct residues modulo $P_{1}$ and in fact all of the rows of $A$ are identical modulo $P_{1}$.

Now let us consider the columns of $A$ modulo L. Set 1.c.m. $\left\{P_{2} f_{2}, \ldots, P_{r} f_{r}\right\}=L f^{\prime}$. From the hypothesis that $(m, f)=1$, it follows easily that $\left(L, f^{\prime}\right)=1$. Thus the induction hypothesis allows us to conclude that $u_{s}, u_{s+f^{\prime}}, \ldots, u_{s+(L-1) f^{\prime}}$ are all distinct modulo $L$. Obviously $L f^{\prime} \mid m f$, thus $f^{\prime} \mid P_{1} f$ and let us write $F f=P_{1} f=h f^{\prime}$. Since $(f, m)=1$, this implies that $(h, L)=1$, thus we may apply Lemma 8 to conclude that each of the entries of any column of $A$ are distinct modulo $L$.

The Chinese Remainder Theorem can now be invoked to conclude that $u_{s}, u_{s+f}, \ldots, u_{s+(m-1) f}$ are all distinct modulo $m$.

Now assume that $(m, f) \neq 1$. Then there exists a smallest $v$ such that $\left(P_{i}, f\right)=1$ for $i<v$ and $\left(P_{v}, f\right) \neq 1$. Set $L=P_{v} \cdots P_{r}$ and $F=m / L$, and consider the matrix $A$ with these new values of $F$ and $L$.

Just as in the preceding case it follows that each row of $A$ represents all of the distinct residues modulo $F$ and that all of the rows are identical modulo $F$.

Thus by the Chinese Remainder Theorem, all of the entries of the matrix are distinct modulo $m$ iff all of the entries in any column (which are constant modulo $F$ ) are distinct modulo $L$. We shall show that the entries in the first column are not distinct modulo $L$.

From the first column of $A$ construct a new matrix

$$
B=\left[\begin{array}{ccc}
u_{s} & u_{s+F f} & \cdots u_{s+\left(P_{v}-1\right) F f} \\
u_{s+P_{v} F f} & \cdots & \\
\vdots & & \\
u_{s+\left(P_{v+1} \cdots P_{r}-1\right) P_{v} F f} & \cdots & u_{s+(L-1) F f}
\end{array}\right] .
$$

Now $P_{v} f_{v} \mid F L f$, so $f_{v} \mid F P_{v+1} \cdots P_{r} f$. Further, since $f_{v} \mid p_{v}-1$ and $p_{v}<p_{v+1}<$ $\cdots<p_{r}$, we have that $\left(f_{v}, P_{v+1} \cdots P_{r}\right)=1$, so $f_{v} \mid F f$, thus the rows are identical modulo $P_{v}$. Set $F f=h f_{v}$. By assumption $p_{v} \mid f$ and since $\left(p_{v}, f_{v}\right)=1$, we must have that $p_{v} \mid h$. However, we can now apply Lemma 8 to conclude that the rows of $B$ are not all distinct modulo $P_{v}$, which in turn implies that the entries of $B$ are not all distinct modulo $L$. 
As we pointed out it has been proved that if $\left(u_{n}\right)$ is $\operatorname{UD}\left(\bmod P_{i}\right), i=1, \ldots, r$, then $\left(u_{n}\right)$ is $\mathrm{UD}(\bmod m)$, where $m=P_{1} \cdots P_{r}$. The proof of Theorem 9 did not need this result and in fact we can use Theorem 9 to prove this result on $\mathrm{UD}(\bmod m)$. We shall not give a complete proof but rather just indicate how Theorem 9 can be used.

Suppose that $\left(u_{n}\right)$ is $\mathrm{UD}\left(\bmod P_{i}\right)$, then by Theorem $\mathrm{B},\left(u_{n}\right)$ is $f_{i}-\mathrm{UD}\left(\bmod P_{i}\right)$, where $f_{i} \mid p_{i}-1$. Let $m f=1$.c.m. $\left\{P_{1} f_{1}, \ldots, P_{r} f_{r}\right\}$. If $(f, m)=1$, then by Theorem 9 , $\left(u_{n}\right)$ is $f-\mathrm{UD}(\bmod m)$ and thus $\left(u_{n}\right)$ is $\mathrm{UD}(\bmod m)$.

If $(f, m) \neq 1$ then there are technical complications. However, if we assume that $P_{i} \neq 2$ or 3 for all $i$, then we can apply Theorem 9 quite easily as the following argument shows.

If $P_{i} \neq 2$ or 3 and $\left(u_{n}\right)$ is $f_{i} \mathrm{UDD}\left(\bmod P_{i}\right)$ then by Theorem $A,\left(u_{n}\right)$ is $f_{i}$ $\mathrm{UD}\left(\bmod P_{i}^{e}\right)$, for all $e$.

Thus since $(m, f) \neq 1$, let $e$ be a sufficiently large integer so that 1.c.m. $\left\{P_{1}^{e} f_{1}, \ldots, P_{r}^{e} f_{r}\right\}=P_{1}^{e} \cdots P_{r}^{e} f^{\prime}=m^{e} f^{\prime}$, where $\left(f^{\prime}, m\right)=1$. Thus by Theorem $9,\left(u_{n}\right)$ is $f^{\prime}-\mathrm{UD}\left(\bmod m^{e}\right)$, so $\left(u_{n}\right)$ is $\operatorname{UD}\left(\bmod m^{e}\right)$ and it then follows that $\left(u_{n}\right)$ is $\mathrm{UD}(\bmod m)$.

If $P_{i}=2$ or 3 for some $i$ then we cannot use the above argument since it is possible that $\left(u_{n}\right)$ is UD modulo 2 or 3 , yet $\left(u_{n}\right)$ is not UD modulo $2^{2}$ or $3^{2}$, respectively.

Thus, if $P_{i}=2$ or 3 then we would set $F=P_{1}, L=P_{2} \cdots P_{r}$ if exactly one of the $P_{i}$ is 2 or 3 and we would set $F=P_{1} P_{2}, L=P_{3} \cdots P_{r}$ if $P_{1}=2, P_{2}=3$.

If $(f, L) \neq 1$ then we can replace $L$ by $L^{e}$ for $e$ sufficiently large (just as in the preceding argument) so that l.c.m. $\left\{F, L^{e}, f_{i}, i=1, \ldots, r\right\}=F L^{e} f^{\prime}$, where $\left(f^{\prime}, L\right)=$ 1. Thus if $\left(u_{n}\right)$ is $\operatorname{UD}\left(\bmod F L^{e}\right)$, then $\left(u_{n}\right)$ is $\operatorname{UD}(\bmod F L)$. So we can assume that $(f, L)=1$, yet $(f, F) \neq 1$; thus there are three possibilities (i) $2|f, 3| f$, (ii) $2 \mid f$, $3+f$, (iii) $2+f, 3 \mid f$. All of these cases entail the same kind of analysis which we will not discuss further.

\section{REFERENCES}

1. Lee Erlebach and William Yslas Vélez, Equiprobability in the Fibonacci sequence, Fibonacci Quart. 21 (1983), 189-191.

2. Whadysław Narkiewicz, Uniform distribution of sequences of integers in residue classes, Lecture Notes in Math., vol. 1087, Springer-Verlag, New York, 1984.

Department of Mathematics, University of Arizona, Tucson, Arizona 85721 